\title{
The selective and non-selective cyclooxygenase inhibitors valdecoxib and piroxicam induce the same postoperative analgesia and control of trismus and swelling after lower third molar removal
}

\author{
V. Benetello ${ }^{1}$, \\ F.C. Sakamoto ${ }^{1}$, \\ F.P.M. Giglio ${ }^{1}$, V.T. Sakai ${ }^{2}$, \\ A.M. Calvo ${ }^{1}$, K.C.S. Modena ${ }^{1}$, \\ B.L. Colombini ${ }^{1}$, \\ T.J. Dionísio' ${ }^{1}$, J.R.P. Lauris², \\ F.A.C. Faria ${ }^{1}$ and C.F. Santos ${ }^{1}$
}

\author{
'Departamento de Ciências Biológicas, ²Departamento de Odontopediatria, \\ Ortodontia e Saúde Coletiva, Faculdade de Odontologia de Bauru, \\ Universidade de São Paulo, Bauru, SP, Brasil
}

\author{
Correspondence \\ C.F. Santos \\ Disciplina de Farmacologia \\ Faculdade de Odontologia de Bauru \\ Universidade de São Paulo \\ Al. Dr. Octávio P. Brisolla, 9-75 \\ 17012-901 Bauru, SP \\ Brasil \\ Fax: +55-14-3223-4679 \\ E-mail: cebola@usp.br \\ V. Benetello and F.C. Sakamoto \\ were recipients of $\mathrm{CNPq}$ \\ (No.103394/2005-2) and FAPESP \\ (No. 04/13335-1) fellowships, \\ respectively.
}

Publication supported by FAPESP (No. 2007/02946-8).

Received November 20, 2006 Accepted April 23, 2007

\begin{abstract}
We compared the clinical efficacy of orally administered valdecoxib and piroxicam for the prevention of pain, trismus and swelling after removal of horizontally and totally intrabony impacted lower third molars. Twenty-five patients were scheduled to undergo removal of symmetrically positioned lower third molars in two separate appointments. Valdecoxib $(40 \mathrm{mg})$ or piroxicam $(20 \mathrm{mg})$ was administered in a double-blind, randomized and crossed manner for 4 days after the surgical procedures. Objective and subjective parameters were recorded for comparison of postoperative courses. Both agents were effective for postoperative pain relief $(\mathrm{N}=19)$. There was a similar mouth opening at suture removal compared with the preoperative values $(86.14 \pm 4.36$ and $93.12 \pm 3.70 \%$ of the initial measure for valdecoxib and piroxicam, respectively; ANOVA). There was no significant difference regarding the total amount of rescue medication taken by the patients treated with valdecoxib or piroxicam (173.08 \pm 91.21 and $461.54 \pm 199.85 \mathrm{mg}$, respectively; Wilcoxon test). There were no significant differences concerning the swelling observed on the second postoperative day compared to baseline measures (6.15 \pm 1.84 and $8.46 \pm 2.04 \mathrm{~mm}$ for valdecoxib and piroxicam, respectively; ANOVA) or on the seventh postoperative day $(1.69 \pm 1.61$ and $2.23 \pm$ $2.09 \mathrm{~mm}$ for valdecoxib and piroxicam, respectively; ANOVA). The cyclooxygenase- 2 selective inhibitor valdecoxib is as effective as the non-selective cyclooxygenase inhibitor piroxicam for pain, trismus and swelling control after removal of horizontally and totally intrabony impacted lower third molars.
\end{abstract}

Key words - Acute postoperative pain - Cyclooxygenase inhibitors

- Lower third molar

- Non-steroidal

anti-inflammatory drugs

- Piroxicam

- Valdecoxib 


\section{Introduction}

Pain as a consequence of lower third molar surgeries has been extensively studied and, therefore, has become a model for the evaluation of the efficacy of many therapeutic approaches in clinical pharmacology. Frequently, this is a short-lasting and moderate pain, reaching its maximum intensity within a short period of time after the end of surgery, and, in most cases, patients require some kind of analgesic to treat it (1). Besides pain, swelling and mouth opening limitation associated with the inflammatory response are also undesirable consequences for patients who undergo surgical interventions in the oral cavity (2).

Several non-steroidal anti-inflammatory drugs (NSAIDS) have been used for pain, trismus and swelling control after lower third molar surgeries (2-6). The therapeutic effect of these medications is based on the inhibition of cyclooxygenases (COX), which determines an inhibition of prostaglandin production (7). However, their use has been associated with a large number of adverse effects, including gastrointestinal ulceration and bleeding, inhibition of platelet aggregation, and alterations in renal function (7-9).

Three COX isoforms are known: COX1 , a constitutive form expressed in almost all tissues, COX-2, which is predominantly induced and constitutively expressed in a limited number of tissues (renal medulla, prostate, brain, and endothelium) $(7,10,11)$, and COX-3, a COX-1-derived protein, most abundantly found in the cerebral cortex and heart (12). COX-2 is believed to be the main isoenzyme for pro-inflammatory prostaglandin production (7). Thus, despite their high cost, COX-2 selective inhibitors such as coxibs have been extensively used in order to selectively inhibit COX-2, but not COX1 , resulting in therapeutic effects comparable with those of conventional NSAIDS, but with less adverse reactions for patients $(7,13)$. It is worth mentioning that recent reports have suggested an increased risk of cardiovascular events for patients taking coxibs. Therefore, clinicians must consider the efficacy, gastrointestinal and cardiovascular risks, concomitant medications and costs when determining the appropriateness of COX-2 selective NSAID therapy (14-19).

Among the NSAIDS used in dentistry, piroxicam, a non-selective COX inhibitor, has been extensively studied (20-24). In contrast, there are only seven reports in the dental literature concerning the clinical use of valdecoxib, a COX-2 selective inhibitor, for postoperative pain control in patients who undergo dental surgeries $(13,25-30)$. In these studies, either different doses of valdecoxib or its injectable prodrug parecoxib were tested $(9,16,17,19)$ or a comparison with rofecoxib, another COX-2 selective inhibitor, was performed $(25,28,30)$. Additionally, only one study compared valdecoxib with a traditional non-selective NSAID (ketorolac), with both agents being parenterally administered (29). To date, no study has compared oral valdecoxib with an orally administered nonselective COX inhibitor. Like valdecoxib, piroxicam is a long-acting NSAID. Due to their similar pharmacokinetic characteristics, both drugs can be administered once daily. Therefore, piroxicam is a suitable drug to be compared with valdecoxib.

Hence, the aim of the present study was to compare the clinical efficacy of orally administered valdecoxib and piroxicam for postoperative pain, trismus and swelling control in lower third molar removal. For this purpose, the experimental model of surgical removal of symmetrically positioned lower third molars was used (4-6).

\section{Material and Methods}

The Ethics Committee of our institution approved the protocol of this study (process \#104/2004). All patients provided written informed consent during the pretreatment 
screening period before any study procedures were performed.

The study population comprised $25 \mathrm{pa}-$ tients aged 18 years or over, with two horizontally and totally intrabony impacted lower third molars, as observed in panoramic radiographies. Eligibility criteria included absence of systemic illness and inflammation or infection at the extraction sites. Exclusion criteria were any history of allergic reaction to local anesthetics, gastrointestinal bleeding or ulceration, cardiovascular and kidney diseases, and allergy to aspirin, piroxicam or any other NSAID. Pregnant women were also excluded from the study. Instructions for not using antidepressants, diuretics or aspirin during the days preceding the surgeries were given to the patients, since these drugs could cause hemorrhage or other blood problems, thus interfering with the results of the investigation (5).

This was a double-blind study, that is, neither the surgeon nor the patients were aware of the anti-inflammatory agent (valdecoxib or piroxicam) being prescribed postoperatively at the two different appointments. Each patient required surgical treatment with the same magnitude of trauma (bone removal and tooth sectioning) on opposite sides of the mandibular jaw, which was performed during two visits 1 to 2 months apart (4-6,31). For postoperative pain relief, in the first appointment, the patients randomly received either valdecoxib or piroxicam. In the second appointment, the NSAID not used previously was then administered in a crossed manner. The same surgeon performed all surgeries and postoperative controls. The patients received a regional anesthetic blockade of buccal, lingual and inferior alveolar nerves with $1.8 \mathrm{~mL} 4 \%$ articaine with $1: 100,000$ adrenaline $(5,6)$. When anesthesia of the lower lip was achieved, an additional $0.9 \mathrm{~mL}$ of the same anesthetic was infiltrated into the mucosa in order to guarantee hemostasis and anesthesia at the site $(5,6)$. The removal of third molars followed a standard surgical technique. Upon completion of the surgeries, the surgical sites were thoroughly irrigated, suctioned, and sutured. Patients remained in the clinic for the first postoperative hour.

The NSAID administration protocol was $40 \mathrm{mg}$ valdecoxib and $20 \mathrm{mg}$ piroxicam once daily for 4 days. Rescue analgesic medication was available to any patient as needed throughout the study; for this purpose, 750 $\mathrm{mg}$ paracetamol was provided to all patients $(5,6)$. Patients recorded the date and time when rescue medication was taken. They were also instructed not to interrupt the use of the anti-inflammatory, even if they had taken rescue analgesic medication.

The following parameters were assessed: duration of the surgery after anesthetic administration (in minutes), which corresponded to the period between the first incision and the last suture $(2,5,6)$; subjective postoperative pain evaluation with the aid of a 100-mm length visual analogue scale, with 0 corresponding to "no pain" and 100 corresponding to "worst pain imaginable" $(5,6,13)$; subjects recorded the intensity of postoperative pain at 15-min intervals for the first 60 postoperative minutes, and $1.5,2,3,4,5,6$, $7,8,10,12,16,24(6,13,25), 48,72$, and 96 $\mathrm{h}$ (6) after the end of surgery; mouth opening (distance, in $\mathrm{mm}$, between the mesial-incisal corners of the upper and lower right central incisors at maximum opening of the jaws) before surgery and at the time of suture removal (7 days postoperatively); the postoperative ability to open the mouth was expressed as a percentage of the preoperative measure $(2,5,6)$; incidence, type and severity of adverse reactions (gastrointestinal irritation, nausea, vomiting, bleeding, allergy, headache, dizziness, sleepiness, and any other kinds of reaction) $(5,6,13)$; total amount of rescue analgesic medication (paracetamol, in $\mathrm{mg}$ ) needed during the postoperative period, and facial swelling determined by a tape-measuring method on the 2nd and 7th postoperative days (2). This method takes 
into account the sum of the following measures: distance between the lateral corner of the eye and angle of the mandible, distance between tragus and outer corner of the mouth, and distance between tragus and soft tissue pogonion. The preoperative sum of the 3 measurements was considered as the baseline for that side. The difference between each postoperative measurement and the

Table 1. Objective and subjective parameters recorded for comparison of postoperative courses after removal, in two separate appointments, of horizontally and totally intrabony impacted lower third molars.

\begin{tabular}{lcc}
\hline Parameter & Valdecoxib & Piroxicam \\
\hline Duration of surgery (min) & $18.51 \pm 2.57$ & $17.78 \pm 3.02$ \\
Total amount of rescue medication $(\mathrm{mg})$ & $173.08 \pm 91.21$ & $461.54 \pm 199.85$ \\
Patients taking rescue medication, N (\%) & $4(30.77 \%)$ & $5(38.46 \%)$ \\
Mouth opening (\%, 7th postoperative day) & $86.14 \pm 4.36$ & $93.12 \pm 3.70$ \\
Swelling (mm, 2nd postoperative day) & $6.15 \pm 1.84$ & $8.46 \pm 2.04$ \\
Swelling (mm, 7th postoperative day) & $1.69 \pm 1.61$ & $2.23 \pm 2.09$ \\
\hline
\end{tabular}

Patients used oral valdecoxib $(40 \mathrm{mg})$ or piroxicam $(20 \mathrm{mg})$ in a double-blind, randomized and crossed manner for 4 days, starting the ingestion immediately after surgery. Data are reported as means \pm SEM for $N=19$ patients unless otherwise indicated. There were no statistically significant differences between treatments when paired $t$ test, Wilcoxon test and two-way ANOVA followed by the Tukey test for multiple comparisons were used.

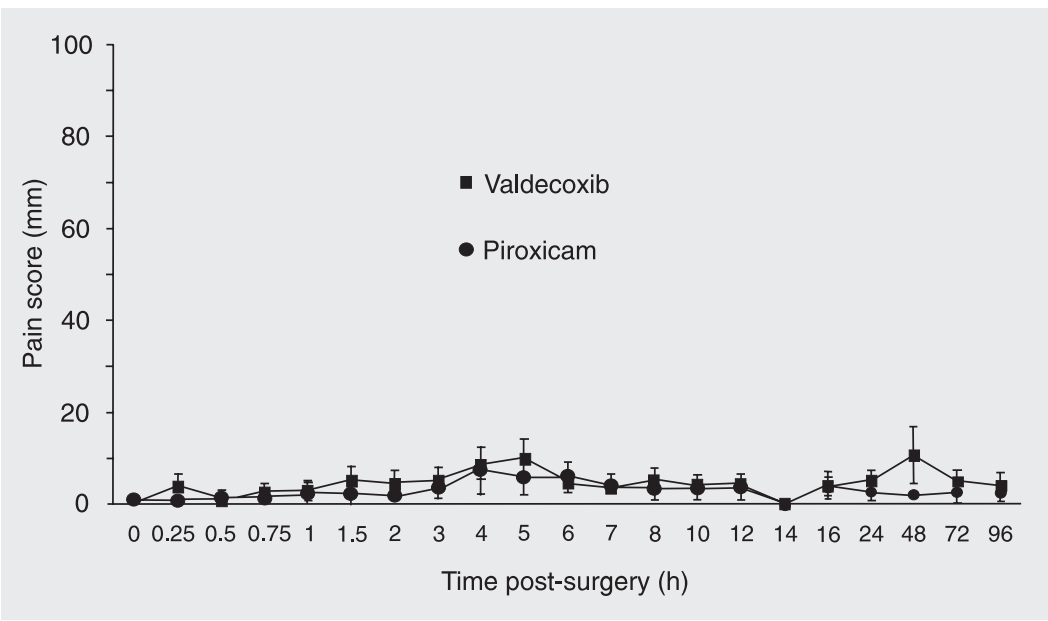

Figure 1. Pain scores (in $\mathrm{mm}$ ) recorded by patients, with the aid of a $100-\mathrm{mm}$ length visual analogue scale (with 0 anchored by "no pain" and 100 anchored by "worst pain imaginable" without full-scale reference point between 0 and 100), at 15-min intervals for the first 60 postoperative min, and 1.5, 2, 3, 4, 5, 6, 7, 8, 10, 12, 16, 24, 48, 72, and $96 \mathrm{~h}$ after removal, in two separate appointments, of horizontally and totally intrabony impacted lower third molars. Patients used oral valdecoxib $(40 \mathrm{mg})$ or piroxicam $(20 \mathrm{mg})$ in a double-blind, randomized and crossed manner for 4 days, starting the ingestion immediately after the surgery. Data reported as means \pm SEM $(N=19)$. baseline indicated the facial swelling for that day.

The paired $t$-test was used to compare the duration of the surgeries. The nonparametric Wilcoxon test was employed to assess the parameters "rescue analgesic medication" and "postoperative pain". Data regarding "mouth opening" and "facial swelling" were submitted to statistical analysis using twoway ANOVA followed by the Tukey test for multiple comparisons. Statistical significance was established at 5\%. Data are reported as means \pm SEM.

\section{Results}

Initially, 25 patients were operated. All of them returned to the clinic on the 7th postoperative day for suture removal after the first surgical procedure. The patients brought the data of subjective pain evaluation during the postoperative period, and the date and time when rescue medication was taken. Nineteen patients ( 9 males and 10 females; mean age 20.61 years) underwent the second surgery before valdecoxib was withdrawn from the market on April 7, 2005. However, the results of pain scores and analgesic intake for the 6 patients who dropped out of the study were closely similar to those for the subjects who finished it (data not shown).

No statistically significant difference in the mean duration of the two surgeries was observed (paired $t$-test; Table 1), which permitted a comparative assessment of the clinical efficacy of both NSAIDS studied.

According to the patients' evaluation on the visual analogue scale, the reported pain scores were low. No statistically significant difference in the analgesia promoted by the two medications occurred during the periods of observation (Wilcoxon test; Figure 1).

There was no statistically significant difference concerning the total amount of rescue analgesic medication (paracetamol) when the patients were treated either with valde- 
coxib or piroxicam $(173.08 \pm 91.21$ and $461.54 \pm 199.85 \mathrm{mg}$, respectively; Wilcoxon test; Table 1). The percentage of patients taking rescue medication was closely similar in both groups (30.77 and $38.46 \%$ for valdecoxib and piroxicam, respectively; Wilcoxon test; Table 1).

There was a similar mouth opening at the time of suture removal compared with the preoperative measures for each patient in both treatment groups $(86.14 \pm 4.36$ and $93.12 \pm 3.70 \%$ of the initial measure for valdecoxib and piroxicam, respectively; ANOVA; Table 1). Besides, no adverse reactions to either medication were observed.

Finally, there were no significant differences concerning the swelling observed on the 2 nd postoperative day in comparison with baseline measures $(6.15 \pm 1.84$ and $8.46 \pm 2.04 \mathrm{~mm}$ for valdecoxib and piroxicam, respectively; ANOVA) or on the seventh postoperative day $(1.69 \pm 1.61$ and 2.23 $\pm 2.09 \mathrm{~mm}$ for valdecoxib and piroxicam, respectively; ANOVA; Table 1).

\section{Discussion}

This study evaluated the clinical efficacy of multiple oral dose regimen of $40 \mathrm{mg}$ valdecoxib and $20 \mathrm{mg}$ piroxicam in patients after aggressive oral surgeries under local anesthesia. The patients were carefully selected in relation to position and bone impaction of both lower third molars, and only those presenting both teeth in a horizontal and totally intrabony position were included. This inclusion criterion reduced the chances of locating many subjects, because it represents a rare condition. Despite these difficulties, the total number of patients in our study is closely similar to that of other reports of lower third molar removal $(5,32)$. The number of patients enrolled in our study, 19, is also justified by the fact that on April 7, 2005, valdecoxib was withdrawn from the market, at least in part, due to cardiovascular risk with chronic use and also serious skin conditions (33). The patients who completed the study underwent both surgical procedures before this date.

Valdecoxib has been assessed in clinical studies of analgesic efficacy after third molar removal $(13,25-30)$. However, none of these studies used the surgical model employed in ours. Desjardins et al. $(26,27)$ evaluated patients who needed surgical removal of two ipsilateral impacted third molars. In other studies, all patients had undergone surgical extraction of 2 or more impacted third molars, one of which was mandibular $(13,29,30)$. Finally, other studies $(25,28)$ assessed patients requiring the removal of three or more third molars, of which at least two were impacted, and at least one was an impacted mandibular molar. Therefore, the lack of uniformity of the methodology employed in these studies impairs the comparison of our results with others.

Dose-response experiments demonstrated that oral $40 \mathrm{mg}(13,27)$ or parenteral (intravenous) 40 and $50 \mathrm{mg}$ valdecoxib $(26,29)$ are effective doses for analgesic purposes in the removal of third molars. A multiple dose regimen was never adopted to test the clinical efficacy of valdecoxib after lower third molar removal. This approach seems more realistic from a clinical point of view than a single-dose regimen, since maximum facial swelling is expected to occur $48 \mathrm{~h}$ after the surgical procedure (34), although it may increase on the third postoperative day (35). Moreover, pain reaches a peak as the anesthetic wears off and the full inflammatory response to surgery evolves (27), and may last for three to five days after the procedure (36). The immediate intake of an analgesic drug is also favorable since waiting for a patient to report severe pain before prescribing an analgesic produces unnecessary suffering and might reduce the efficacy of any subsequent treatment (27).

Low pain complaint was observed during both postoperative periods, either with 
the use of $20 \mathrm{mg}$ piroxicam or $40 \mathrm{mg}$ valdecoxib. These findings agree with those of Desjardins (20) and Daniels et al. (13). Thus, a multiple dose regimen with both NSAIDS provided a sustained level of analgesia over the evaluation period, with no statistically significant difference between the two drugs (Figure 1). The percentage of patients taking rescue medication was also very similar to that reported in another study assessing the clinical efficacy of valdecoxib, that is, about $32 \%$ (27). These results confirm the analgesic efficacy of piroxicam and valdecoxib after lower third molar removal $(13,20-29)$.

In the current study, we performed a further characterization of the clinical efficacy of both valdecoxib and piroxicam focusing not only on postoperative pain relief (13,23-29), but also on trismus and swelling control. Both drugs were equally effective for trismus control. The measurements of facial swelling found in the study of Üstün et al. (2), who prescribed two different doses of a corticosteroid for swelling control, were very similar to ours both on the 2nd and 7th postoperative days (about 7 and $3 \mathrm{~mm}$, respectively). Corticosteroids are well known as potent drugs widely used to decrease the swelling related to third molar surgery $(2,24)$.

No clinically significant adverse reaction was observed in our study, confirming that valdecoxib $(13,25,27)$ and piroxicam (20-22) are often well tolerated by patients with pain complaints after dental surgeries. It is widely known that COX-2 selective inhibitors are associated with decreased adverse gastrointestinal complications as compared with conventional NSAIDS. However, taking into account the short-term treatment with valdecoxib and piroxicam (4 days), both drugs were safe for our patients. Piroxicam requires more than 1 month to induce deleterious gastrointestinal side effects, with a maximum risk at 50 days (37).
Although valdecoxib was withdrawn from the market, other coxibs are still commercially available: celecoxib, etoricoxib and lumiracoxib. These agents have shown good analgesic efficacy after third molar removal $(4,38-40)$, hence it is important to continue with studies on the effect of COX-2 selective inhibitors in general. As pointed out by Hur et al. (18), the current data suggest that coxibs carry different risk of cardiovascular events and that the risk may be dose related. The clinician's choice of a particular drug of this group at a lower dose may benefit an individual who has a relatively low cardiovascular risk but a high risk for gastrointestinal complications. Additionally, for patients who do not obtain proper pain control with a nonselective NSAID but find that coxibs are more effective, this added benefit should be considered along with the possible risks (18).

Finally, the COX-2 selective inhibitor valdecoxib and the conventional NSAID piroxicam are equally effective and well tolerated by patients when administered in a multiple oral dose regimen for pain, trismus and swelling control after removal of horizontally and totally intrabony impacted lower third molars. Taking into account the high cost of COX-2 selective inhibitors $(17,18)$, such as valdecoxib, in comparison with the conventional NSAIDS like piroxicam, our data support the suggestion that there are no sufficient clinical advantages for the prescription of valdecoxib after lower third molar removal.

\section{Acknowledgments}

The authors would like to thank Vera Lúcia Rufino Rosa for excellent secretarial assistance. We also thank Nereu Daltin Junior and Simone Lira Mendes, Laboratórios Pfizer Ltda., for donating piroxicam. 


\section{References}

1. McGrath C, Comfort MB, Lo EC, Luo Y. Changes in life quality following third molar surgery - the immediate postoperative period. Br Dent J 2003; 194: 265-268.

2. Üstün $Y$, Erdogan O, Esen E, Karsli ED. Comparison of the effects of 2 doses of methylprednisolone on pain, swelling, and trismus after third molar surgery. Oral Surg Oral Med Oral Pathol Oral Radiol Endod 2003; 96: 535-539.

3. Barden J, Edwards JE, McQuay HJ, Wiffen PJ, Moore RA. Relative efficacy of oral analgesics after third molar extraction. Br Dent $J$ 2004; 197: 407-411.

4. Calvo AM, Sakai VT, Modena KCS, Colombini BL, Gallina MC, Dionísio TJ, et al. Comparison of the efficacy of etoricoxib and ibuprofen in pain and trismus control after lower third molar removal. Rev Odontol UNICID 2006; 18: 29-36.

5. Colombini BL, Modena KC, Calvo AM, Sakai VT, Giglio FP, Dionisio $\mathrm{TJ}$, et al. Articaine and mepivacaine efficacy in postoperative analgesia for lower third molar removal: a double-blind, randomized, crossover study. Oral Surg Oral Med Oral Pathol Oral Radiol Endod 2006; 102: 169-174.

6. Calvo AM, Sakai VT, Giglio FP, Modena KC, Colombini BL, Benetello $V$, et al. Analgesic and anti-inflammatory dose-response relationship of 7.5 and $15 \mathrm{mg}$ meloxicam after lower third molar removal: a double-blind, randomized, crossover study. Int J Oral Maxillofac Surg 2007; 36: 26-31.

7. Lee $\mathrm{Y}$, Rodriguez C, Dionne RA. The role of COX-2 in acute pain and the use of selective COX-2 inhibitors for acute pain relief. Curr Pharm Des 2005; 11: 1737-1755.

8. Baker DE. Valdecoxib. Rev Gastroenterol Disord 2002; 2: 116-125.

9. Chavez ML, DeKorte CJ. Valdecoxib: a review. Clin Ther 2003; 25: 817-851.

10. Alsalameh S, Burian M, Mahr G, Woodcock BG, Geisslinger G. Review article: The pharmacological properties and clinical use of valdecoxib, a new cyclo-oxygenase-2-selective inhibitor. Aliment Pharmacol Ther 2003; 17: 489-501.

11. Patrignani $\mathrm{P}$, Capone ML, Tacconelli S. Clinical pharmacology of etoricoxib: a novel selective COX2 inhibitor. Expert Opin Pharmacother 2003; 4: 265-284.

12. Chandrasekharan NV, Dai H, Roos KL, Evanson NK, Tomsik J, Elton TS, et al. COX-3, a cyclooxygenase-1 variant inhibited by acetaminophen and other analgesic/antipyretic drugs: cloning, structure, and expression. Proc Natl Acad Sci U S A 2002; 99: 1392613931.

13. Daniels SE, Desjardins PJ, Talwalker S, Recker DP, Verburg KM. The analgesic efficacy of valdecoxib vs. oxycodone/acetaminophen after oral surgery. J Am Dent Assoc 2002; 133: 611-621.

14. Levesque LE, Brophy JM, Zhang B. The risk for myocardial infarction with cyclooxygenase-2 inhibitors: a population study of elderly adults. Ann Intern Med 2005; 142: 481-489.

15. Levesque LE, Brophy JM, Zhang B. Time variations in the risk of myocardial infarction among elderly users of COX-2 inhibitors. CMAJ 2006; 174: 1563-1569.

16. Tannenbaum H, Bombardier C, Davis P, Russell AS. An evidencebased approach to prescribing nonsteroidal antiinflammatory drugs. Third Canadian Consensus Conference. J Rheumatol 2006; 33: 140-157.

17. Chaiamnuay S, Allison JJ, Curtis JR. Risks versus benefits of cyclooxygenase-2-selective nonsteroidal antiinflammatory drugs. Am J Health Syst Pharm 2006; 63: 1837-1851.

18. Hur C, Chan AT, Tramontano AC, Gazelle GS. Coxibs versus com- bination NSAID and PPI therapy for chronic pain: an exploration of the risks, benefits, and costs. Ann Pharmacother 2006; 40: 10521063.

19. Zarraga IG, Schwarz ER. Coxibs and heart disease: what we have learned and what else we need to know. J Am Coll Cardiol 2007; 49: 1-14.

20. Desjardins PJ. Analgesic efficacy of piroxicam in postoperative dental pain. Am J Med 1988; 84: 35-41.

21. Graziani F, Corsi L, Fornai M, Antonioli L, Tonelli M, Cei S, et al. Clinical evaluation of piroxicam-FDDF and azithromycin in the prevention of complications associated with impacted lower third molar extraction. Pharmacol Res 2005; 52: 485-490.

22. Selcuk E, Gomel M, Apaydin S, Kose T, Tuglular I. The postoperative analgesic efficacy and safety of piroxicam (FDDF) and naproxen sodium. Int J Clin Pharmacol Res 1998; 18: 21-29.

23. Alpaslan C, Alpaslan G, Ugar D. Postoperative pain control by single doses of piroxicam administered sublingually and aspirin. $J$ Marmara Univ Dent Fac 1997; 2: 658-664.

24. Barroso AB, Lima V, Guzzo GC, Moraes RA, Vasconcellos MC, Bezerra MM, et al. Efficacy and safety of combined piroxicam, dexamethasone, orphenadrine, and cyanocobalamin treatment in mandibular molar surgery. Braz J Med Biol Res 2006; 39: 12411247.

25. Christensen KS, Cawkwell GD. Valdecoxib versus rofecoxib in acute postsurgical pain: results of a randomized controlled trial. J Pain Symptom Manage 2004; 27: 460-470.

26. Desjardins PJ, Grossman EH, Kuss ME, Talwalker S, Dhadda S, Baum D, et al. The injectable cyclooxygenase-2-specific inhibitor parecoxib sodium has analgesic efficacy when administered preoperatively. Anesth Analg 2001; 93: 721-727.

27. Desjardins PJ, Shu VS, Recker DP, Verburg KM, Woolf CJ. A single preoperative oral dose of valdecoxib, a new cyclooxygenase-2 specific inhibitor, relieves post-oral surgery or bunionectomy pain. Anesthesiology 2002; 97: 565-573.

28. Fricke J, Varkalis J, Zwillich S, Adler R, Forester E, Recker DP, et al. Valdecoxib is more efficacious than rofecoxib in relieving pain associated with oral surgery. Am J Ther 2002; 9: 89-97.

29. Mehlisch DR, Desjardins PJ, Daniels S, Hubbard RC. Single doses of parecoxib sodium intravenously are as effective as ketorolac in reducing pain after oral surgery. J Oral Maxillofac Surg 2003; 61: 1030-1037.

30. Daniels SE, Desjardins PJ, Bird SR, Smugar SS, Tershakovec AM. Rofecoxib $50 \mathrm{mg}$ and valdecoxib 20 or $40 \mathrm{mg}$ in adults and adolescents with postoperative pain after third molar extraction: results of two randomized, double-blind, placebo-controlled, single-dose studies. Clin Ther 2006; 28: 1022-1034.

31. Meechan JG, Cole B, Welbury RR. The influence of two different dental local anaesthetic solutions on the haemodynamic responses of children undergoing restorative dentistry: a randomised, singleblind, split-mouth study. Br Dent J 2001; 190: 502-504.

32. Presser Lima PV, Fontanella V. Analgesic efficacy of aceclofenac after surgical extraction of impacted lower third molars. Int $J$ Oral Maxillofac Surg 2006; 35: 518-521.

33. Brophy JM. Celecoxib and cardiovascular risks. Expert Opin Drug Saf 2005; 4: 1005-1015.

34. Troullos ES, Hargreaves KM, Butler DP, Dionne RA. Comparison of nonsteroidal anti-inflammatory drugs, ibuprofen and flurbiprofen, with methylprednisolone and placebo for acute pain, swelling, and trismus. J Oral Maxillofac Surg 1990; 48: 945-952. 
35. Milles M, Desjardins PJ. Reduction of postoperative facial swelling by low-dose methylprednisolone: an experimental study. J Oral Maxillofac Surg 1993; 51: 987-991.

36. Wynn RL. Update on nonprescription pain relievers for dental pain. Gen Dent 2004; 52: 94-98.

37. Richy F, Bruyere O, Ethgen O, Rabenda V, Bouvenot G, Audran M, et al. Time dependent risk of gastrointestinal complications induced by non-steroidal anti-inflammatory drug use: a consensus statement using a meta-analytic approach. Ann Rheum Dis 2004; 63: 759-766.
38. Khan AA, Brahim JS, Rowan JS, Dionne RA. In vivo selectivity of a selective cyclooxygenase 2 inhibitor in the oral surgery model. Clin Pharmacol Ther 2002; 72: 44-49.

39. Kellstein D, Ott D, Jayawardene S, Fricke J. Analgesic efficacy of a single dose of lumiracoxib compared with rofecoxib, celecoxib and placebo in the treatment of post-operative dental pain. Int $J$ Clin Pract 2004; 58: 244-250.

40. Capone ML, Tacconelli S, Patrignani P. Clinical pharmacology of etoricoxib. Expert Opin Drug Metab Toxicol 2005; 1: 269-282. 